\title{
Efecto de la Incorporación de Plasma Sanguíneo y Pasta de Ajonjolí en la Fabricación de un Embutido tipo Salchicha
}

\author{
Piedad M. Montero*, Diofanor Acevedo, Arnulfo J. Arnedo y Nayelis K. Miranda \\ Universidad de Cartagena, Facultad de Ingeniería, Programa de ingeniería de Alimentos, Grupo de \\ Investigación NUSCA, Avenida el Consulado, Calle 30 No. 48-152. Cartagena, Bolívar-Colombia \\ (e-mail: pmargaritamontero@hotmail.com) \\ * autor a quien debe ser dirigida la correspondencia
}

Recibido Feb. 4, 2015; Aceptado May. 3, 2015; Versión final Jul. 9, 2015, Publicado Dic. 2015

\begin{abstract}
Resumen
En la presente investigación se evaluó el efecto de la incorporación de pasta de ajonjolí y plasma sanguíneo de bovino en un producto cárnico tipo salchicha, como sustituto parcial de grasa y de agua respectivamente. Para ello se evaluó la composición proximal, el perfil de ácidos grasos, el rendimiento por cocción, el perfil de textura y la aceptabilidad de la salchicha. Se probaron cinco tratamientos. Los resultados mostraron que el contenido de humedad de los productos formulados con pasta de ajonjolí y plasma sanguíneo de bovino incrementó, en comparación con el tratamiento control. De esta investigación se puede concluir que la incorporación de pasta de ajonjolí y plasma sanguíneo de bovino incrementa la humedad, el contenido proteico y el rendimiento por cocción, sin que ello tenga un efecto negativo en la aceptabilidad.
\end{abstract}

Palabras clave: pasta de ajonjolí, plasma sanguíneo, salchicha, sustituto parcial

\section{Effect of the Addition of Sesame Paste and Bovine Blood Plasma in the Development of a Meat Product Type Sausage}

\begin{abstract}
In this research the effect of the addition of sesame paste and bovine blood plasma in a sausage meat product type, as a partial substitute for fat and water respectively was evaluated. For this, the proximate composition, the fatty acid profile, the performance by cooking, the texture profile and the acceptability of the sausage were evaluated. Five treatments were tested. The results showed that the moisture content of the products formulated with sesame paste and bovine blood plasma increased, compared to the control treatment. From this research it can be concluded that the incorporation of sesame paste and bovine blood plasma increases moisture, protein content and performance by cooking, without having a negative effect on acceptability.
\end{abstract}

Keywords: sesame paste, blood plasma, sausage, partial substitute 


\section{INTRODUCCIÓN}

Investigaciones recientes han recomendado reducir el porcentaje de calorías provenientes de grasas saturadas a niveles inferiores del 30\% con menos del 10\% de calorías provenientes de grasas (García et al., 2012). Es así, como la reducción de grasas saturadas es una de las tendencias más fuertes en el desarrollo de los productos alimenticios de hoy (Schmiele et al., 2015). Cumpliendo con estas nuevas disposiciones, la industria de alimentos ha generado una nueva línea de productos llamados sustitutos de grasa (SDG) que son una gran variedad de compuestos que se utilizan para reemplazar total o parcialmente la grasa empleada en la elaboración de éstos productos; estos sustitutos provienen de proteínas, carbohidratos, grasas modificadas y sintéticas, para la fabricación de alimentos con bajo contenido calórico, además mantienen muchas de las características de textura y sabor que ésta aporta (Feng et al., 2013). Disminuir el consumo de grasa en la dieta es necesaria ya que muchas investigaciones la correlacionan con enfermedades crónicas como las enfermedades cardiovasculares (principalmente grasas saturadas y colesterol), cáncer, la diabetes tipo 2, obesidad, entre otros (Schmiele et al., 2015; Decker y Park, 2010).

La necesidad del consumo de proteína animal ha incentivado la búsqueda de fuentes alternas, capaces de ofrecer alimentos altamente proteicos con cualidades organolépticas aceptables, de allí que las investigaciones apunten hacia el desarrollo de nuevos productos no convencionales para ser utilizados en la alimentación humana (Benítez et al., 2002). La sangre de algunos animales como el bovino, es aprovechada en diversos países para la alimentación humana, incorporada en los alimentos como fuente de proteína de bajo costo, las cuales poseen propiedades funcionales de aplicación en la industria de alimentos (Camacho et al., 2014). La porción liquida de la sangre es conocida como plasma sanguíneo, que se caracteriza por ser más densa que el agua, con un $\mathrm{pH}$ de 7,4 , compuesta por un $90 \%$ en agua, entre un 15 y $20 \%$ en proteína, y finalmente de nutrimentos como vitaminas, minerales y ácidos grasos (Isaza et al., 2010). Las proteínas plásmicas presentan características favorables para su utilización en la industria de los alimentos, dentro de estas encontramos su alto valor nutritivo, su participación como agente emulsificante, espumante y ligante y su capacidad de formar geles y aumentar la rentabilidad (Camacho et al., 2014). Recientemente, las proteínas del plasma de bovino están siendo utilizadas por la industria de productos cárnicos, debido a sus excelentes propiedades funcionales, en especial su capacidad gelificante y de retención de agua en los productos cocidos, mejorando el rendimiento del producto final (Camacho et al., 2014; Isaza et al., 2010).

Por su parte, la semilla de ajonjolí o sésamo ostenta un alto valor nutritivo en proteínas con una composición del 17 a $23 \%$, en lípidos entre 42 a $50 \%$ en ácidos grasos monoinsaturados como el oleico y poliinsaturados como el linoléico, minerales como el calcio, potasio y el hierro y a nivel de vitaminas se encuentran la $\mathrm{E}, \mathrm{K}, \mathrm{B} 3$, B5, B6, el ácido, fólico, biotina, inositol y colina (Julio et al., 2015; Chiriboga, 2013). A partir de la semilla de ajonjolí se puede obtener una pasta usando procesos tecnológicos entre los principales se encuentran el descascarillado, acondicionamiento, la trituración y molienda. La pasta de ajonjolí se destaca como sustituto no cárnico al ser una reciente alternativa en la reducción de grasa animal en productos cárnicos por la calidad de sus aceites que tiene (Acevedo et al., 2014; 2013). Julio et al., (2015) evaluaron la calidad y aceptabilidad de productos cárnicos tipo chorizos formulados con plasma sanguíneo bovino y pasta de ajonjolí, observando que es posible reemplazar porcentajes de agua por plasma sanguíneo bovino y tocino por pasta de ajonjolí en la formulación de chorizos, para mejorar el rendimiento, aumentar el contenido proteico y reducir el contenido de grasa, sin que ello tenga un efecto negativo en la aceptabilidad. De esta forma gran cantidad de autores señalan la importancia de identificar y utilizar sustitutos grasos que ayuden a mejorar los productos cárnicos (Julio et al., 2015; Isaza et al., 2010; Benítez et al., 2011; Rodríguez et al., 2011; Figueroa et al., 2012; Gómez et al., 2013; Camacho et al., 2014). El plasma sanguíneo de bovino es aprovechado en diversos países para la alimentación humana, incorporándolo en los alimentos como fuente de proteína de bajo costo (Parés et al., 2011; Hurtado et al., 2011; Isaza et al., 2010). A pesar de que se encuentran investigaciones sobre el uso del plasma bovino, son pocas las publicadas en relación al uso de la pasta de ajonjolí como sustituto no carnico.

Actualmente, se ha venido acrecentamiento la utilización de plasma sanguíneo, esto es debido a las aprovechamiento que tienen las proteínas plásmicas (Julio et al., 2015; Isaza et al., 2010; Benítez et al., 2011; Rodríguez et al., 2011; Figueroa et al., 2012; Gómez et al., 2013; Camacho et al., 2014). Además de tener estas macromoléculas una aplicación de suma relevancia en la industria cárnica, aportan un sentido ecológico al reducir los problemas de contaminación ambiental referentes a la descarga de la sangre a la interperie por parte de las plantas de beneficio (Figueroa et al., 2012; Gómez et al., 2013). En la presente investigación se evaluó el efecto de la incorporación de pasta de ajonjolí (Sesamum indicum L.) y plasma sanguíneo de bovino en un producto cárnico tipo salchicha, como sustituto parcial de grasa y de agua respectivamente; con el objetivo de 
evaluar la composición proximal, el perfil de ácidos grasos, el rendimiento por cocción, el perfil de textura y la aceptabilidad de la salchicha.

\section{MATERIALES Y MÉTODOS}

\section{Materias primas}

Se utilizó ajonjolí (S. indicum L.) del municipio de Córdoba, Bolívar (Colombia), para la obtención de la pasta de ajonjolí. La sangre de bovino fue suministrada por la planta de sacrificio del municipio de Arjona, Bolívar, para la obtención del plasma sanguíneo. Las materias primas cárnicas y demás insumos fueron obtenidos de distribuidoras de insumos químicos y supermercados de la ciudad de Cartagena, Colombia.

\section{Obtención de la sangre y plasma sanguíneo}

La sangre de bovino se obtuvo de la planta de sacrificio del municipio de Arjona-Bolívar; la sangre fue recolectada en un recipiente de vidrio de $3 \mathrm{~L}$, se depositaron $12 \mathrm{~g}$ del anticoagulante (citrato de sodio); es decir, $4 \mathrm{~g}$ por cada litro de sangre recolectada quedando con una concentración aproximada de $0,4 \% \mathrm{p} / \mathrm{v}$. Luego de la insensibilización o aturdimiento del animal la sangre fue recolectada directamente de la vena yugular en el recipiente. Posteriormente, el frasco con la sangre recolectada se refrigeró a $4^{\circ} \mathrm{C}$, mediante el uso de una nevera con hielo y transportada al laboratorio de Medicina de la Universidad de Cartagena, en donde la sangre se sometió a separación por centrifugación a 3000 rpm durante 10 min en un equipo marca, Kubota modelo 1200. El plasma fue conservado en refrigeración a $4^{\circ} \mathrm{C}$ (Camacho et al., 2014).

\section{Elaboración tecnológica de la pasta untable}

Se utilizó la metodología reportada por Acevedo et al., (2013), en su trabajo de evaluación de las propiedades reológicas de pastas de ajonjolí artesanal y tecnificada. Para la producción de la pasta las semillas de sésamo se descascararon como se detalla a continuación: se tomaron 1000 gramos de semillas a los cuales se les realizo un proceso de limpieza para eliminar impurezas, luego se sumergieron en 6 litros de agua $\left(T=18 \pm 2^{\circ} \mathrm{C}\right)$ durante 12 horas. Las semillas remojadas fueron exprimidas y descascaradas mecánicamente empleando un paño seco para frotar y así desprender la cascara de esta. Las semillas luego se sumergieron durante cinco minutos en una solución salina al 23\%p/p (cada 1000 gramos en 8 litros de solución). En aras de separar las cascaras y otros elementos extraños, la inmersión de las semillas en la solución salina se repitió tres veces, luego fueron tomadas desde la superficie de la solución y lavadas con agua cinco (5) veces para eliminar la sal. Posteriormente las semillas se exprimieron para reducir el contenido de agua de la superficie de las mismas y fueron utilizadas para el proceso de tostado en este estudio. Doscientos cincuenta gramos de estas semillas descortezadas húmedas fueron tostadas a temperaturas de $130^{\circ} \mathrm{C}$ en un horno compacto (marca: Challerger, serie 02451 ) durante 30 minutos con agitación. Se equilibró de inmediato a temperatura ambiente $\left(20^{\circ} \mathrm{C}\right)$ para evitar un calentamiento adicional. Las semillas tostadas se molieron en un molino para grano marca corona, la molienda se realizó dos veces. La pasta producto del molido fue mezclada con $0,10 \%$ de sal y homogenizada en un procesador (marca: Oster), por dos (2) minutos; obteniendo un producto con consistencia cremosa y sabor definido. El producto obtenido fue envasado en recipientes de vidrio cerrados herméticamente y se le aplicó un tratamiento de pasteurización a $95^{\circ} \mathrm{C}$ por 10 minutos. Finalmente la pasta fue almacenada a temperatura de refrigeración.

\section{Elaboración de la salchicha}

Para la preparación de la salchicha se mezcló la carne molida de cerdo y res, el hielo y el plasma para obtener una pasta. La mezcla se sometió a un proceso de curado (sal común y sal nitrada) por un tiempo de 30 minutos a una temperatura de refrigeración de $4^{\circ} \mathrm{C}$. Esta pasta se agregó al cúter con el resto de ingredientes para obtener una pasta fina y con buena emulsión, evitando su rompimiento. La pasta fue embutida en fundas sintéticas resistentes y escaldadas en agua hasta que la temperatura interna de $75^{\circ} \mathrm{C}$. Posteriormente se realizó un choque térmico en agua de hielo a una temperatura de $4^{\circ} \mathrm{C}$. Las muestras así preparadas se almacenaron en refrigeración a $4^{\circ} \mathrm{C}$, hasta llevar a cabo los análisis correspondientes (Hernández y Güemes, 2010).

Formulaciones, análisis y rendimiento por cocción

Se formularon 4 tratamientos con adición de pasta de ajonjolí y plasma sanguíneo de bovino y un tratamiento (T5) o control, cada tratamiento se elaboró dos veces, los ingredientes utilizados se muestran en la Tabla 1 . Se establecieron los porcentajes de plasma sanguíneo bovino y pasta de ajonjolí teniendo en cuenta que no alteraran las propiedades sensoriales de las muestras. Se determinó la humedad, proteínas y contenido graso mediante A.O.A.C. (2003) de las cinco formulaciones. Para la determinación del perfil de ácidos grasos se 
utilizó el método reportado por Zamorano et al., (2007), el cual se realiza mediante cromatografía gaseosa en un equipo Perkin Elmer equipado con un detector de ionización de llama. Para determinar el rendimiento de las muestras se utilizó la metodología reportada por Piñero et al., (2004), donde se pesó la salchicha antes y después de ser cocida y se utilizó la Ecuación (1).

$\%$ de rendimiento $=\frac{\text { peso de la salchicha cocida }}{\text { peso de la salchicha cruda }} * 100$

Tabla 1: Formulación de salchichas

\begin{tabular}{|c|c|c|c|c|c|}
\hline \multirow{2}{*}{ Materias primas } & \multicolumn{5}{|c|}{ Tratamientos } \\
\cline { 2 - 6 } & $\mathrm{T} 1$ & $\mathrm{~T} 2$ & $\mathrm{~T} 3$ & $\mathrm{~T} 4$ & T5 (control) \\
\hline Carne magra de res (\%) & 35 & 35 & 35 & 35 & 35 \\
\hline Carne magra de cerdo (\%) & 30 & 30 & 30 & 30 & 30 \\
\hline Grasa de cerdo (\%) & 13,5 & 13,5 & 12 & 12 & $15 \%$ \\
\hline Pasta de ajonjolí (\%) & 1,5 & 1,5 & 3,0 & 3,0 & - \\
\hline Hielo en escarcha (\%) & 16 & 18 & 18 & 16 & 20 \\
\hline Plasma sanguíneo (\%) & 4,0 & 2,0 & 2,0 & 4,0 & - \\
\hline Peso total masa (\%) & 100 & 100 & 100 & 100 & 100 \\
\hline Sal nitrada (\%) & 0,3 & 0,3, & 0,3 & 0,3 & 0,3 \\
\hline Condimento unipack para salchicha (\%) & 0,6 & 0,6 & 0,6 & 0,6 & 0,6 \\
\hline Polifosfatos (\%) & 4,0 & 4,0 & 4,0 & 4,0 & 1,2 \\
\hline Extendedor para salchichas (\%) & 3 & 3 & 3 & 3 & 3 \\
\hline
\end{tabular}

Análisis de perfil de textura

De acuerdo al método implementado por Hleap y Velasco (2010), se cortaron rodajas de salchicha de aproximadamente $1,5 \mathrm{~cm}$ de grosor y se dejaron reposar por una hora a temperatura ambiente dentro de una bolsa de polietileno para evitar la pérdida de humedad. Se realizó una doble compresión uniaxial a $75 \%$ de deformación (estrés normal) y a una velocidad de cabeza de $1 \mathrm{~mm} / \mathrm{s}$ con un tiempo de espera de 5 segundos entre las compresiones. Se utilizó un Texturómetro marca TA.TX2 $i^{\circledR}$ Plus Stable Micro System, acoplado el programa Texture Expert Exceed versión 2.64. A partir de los datos arrojados por el software se obtuvieron los siguientes parámetros texturales: dureza $\left(\mathrm{kg} \mathrm{m} \mathrm{s}^{-2}\right)$, adhesividad $\left(\mathrm{kg} \mathrm{m}^{2} \mathrm{~s}^{-2}\right)$, cohesividad (adimensional), elasticidad (adimensional) y masticabilidad $(\mathrm{kg})$.

\section{Aceptabilidad}

Para evaluar la aceptabilidad de los productos se empleó un panel de degustación no entrenado, constituido por 40 jóvenes universitarios de ambos sexos. Se utilizó una escala hedónica de seis puntos con las calificaciones de Me gusta mucho (6), Me gusta (5), Me gusta ligeramente (4), Ni me gusta ni me disgusta (3), Me disgusta ligeramente (2) y Me disgusta mucho (1). Se midió el grado de satisfacción que produjo cada muestra al ser degustada por los panelistas, determinando así, el grado de aceptabilidad de cada formulación (Márquez et al., 2006).

\section{Análisis de datos}

Los datos obtenidos en las diferentes determinaciones se analizaron estadísticamente mediante análisis de varianza de una vía (ANOVA), con un nivel de significancia de 0,05; mientras que las medias fueron comparadas usando la Prueba de Múltiples Rangos de Tukey HSD, con un nivel de significancia de 0,05, Todos los análisis fueron realizados mediante el paquete estadístico Statgraphics ${ }^{\circledR}$ Centurion XV (Versión 15.2.06).

\section{ANÁLISIS Y DISCUSIÓN}

\section{Composición proximal}

La composición proximal de los diferentes tratamientos se presenta en la Tabla 2. El contenido de humedad de los productos formulados con pasta de ajonjolí (A) y plasma sanguíneo de bovino (PS) incrementó en comparación con el tratamiento control (T5), sin embargo los tratamientos T2 (1,5\% de PA y $2 \%$ de PS) y T3 (3\% de PA y $2 \%$ de PS) no presentaron diferencias estadísticamente significativas con $T 5(p<0,05)$. El 
tratamiento con mayor contenido de humedad fue T1, el cual tenía el menor porcentaje de PA (1,5\%) y mayor contenido de PS (4\%). Lo anterior es debido a la capacidad de retención de agua que conceden las proteínas del plasma (Rodríguez et al., 2011; Julio et al., 2014), y al contenido de fibra insoluble presente en la pasta de ajonjolí aproximadamente de un $7,5 \%$, que también absorbe parte de ella proporcionando estabilidad a la emulsión cárnica. En el contenido proteico de los diferentes tratamientos se observa que el T4 (3\% de PA y $4 \%$ de PS) presenta mayor contenido de proteína que los demás tratamientos, esto posiblemente debido a que era el producto que contenía mayor adicción de PA y PS de bovino. Por otra parte el T5 (control) mostró menor contenido de proteína, tal como se presenta en la Tabla 2. Estos resultados de composición proximal (humedad, proteína y grasa) concuerda con la investigación de Ruiz y pacheco (2007), quienes evaluaron la composición proximal de salchicha bajas en grasa formuladas con inulina como sustituto parcial de grasa y se observó un comportamiento similar en la cual el contenido de humedad y proteína aumentó con la adición de este y el contenido de grasa disminuyó. Por su parte Gok et al., (2011) evaluaron el efecto de la utilización de semilla de amapola como sustituto de grasa en la formulación de hamburguesa de carne, y los resultados arrojaron que el uso de semillas de amapola en la formulación de las hamburguesas de carne aumentaban el contenido de humedad y disminuía el contenido graso. Las diferencias con otros estudios pueden ser debidas al método, formulación y producto usados (Tirado et al., 2013). En la Tabla 2 medias con diferentes superíndices ( $a, b$ y c) en la misma fila difieren significativamente $(p<0,05)$, De igual forma $T 1=1,5 \%$ de $A, 13,5 \%$ de grasa, $4 \%$ de PS y $16 \%$ de agua; T2= $1,5 \%$ de $A, 13,5 \%$ de grasa, $2 \%$ de PS y $18 \%$ de agua; $T 3=3 \%$ de $A, 12 \%$ de grasa, $2 \%$ de PS y $18 \%$ de agua; T4=3\% de A, $12 \%$ de grasa, $4 \%$ de PS y $16 \%$ de agua); y es T5 (control)= $15 \%$ de grasa y $20 \%$ de agua).

Tabla 2. Composición proximal de los diferentes tratamientos

\begin{tabular}{|c|c|c|c|c|c|}
\hline \multirow{2}{*}{ Parámetro } & \multicolumn{5}{|c|}{ Tratamiento } \\
\cline { 2 - 6 } & $\mathrm{T} 1$ & $\mathrm{~T} 2$ & T3 & T4 & T5 \\
\hline Humedad (\%) & $68,38 \pm 0,539^{\mathrm{b}}$ & $67,98 \pm 0,056^{\mathrm{a}}$ & $67,42 \pm 0,268^{\mathrm{a}}$ & $68,18 \pm 0,041^{\mathrm{b}}$ & $67,03 \pm 0,030^{\mathrm{a}}$ \\
\hline Proteína (\%) & $19,62 \pm 0,090^{\mathrm{a}}$ & $19,43 \pm 0,128^{\mathrm{a}}$ & $18,74 \pm 0,062^{\mathrm{b}}$ & $19,76 \pm 0,307^{\mathrm{a}}$ & $18,66 \pm 0,040^{\mathrm{b}}$ \\
\hline Grasa (\%) & $15,13 \pm 0,072^{\mathrm{b}}$ & $15,30 \pm 0,020^{\mathrm{b}}$ & $14,50 \pm 0,025^{\mathrm{a}}$ & $14,59 \pm 0,007^{\mathrm{a}}$ & $18,40 \pm 0,020^{\mathrm{c}}$ \\
\hline
\end{tabular}

Composición de análisis de perfil de ácidos grasos

La composición de ácidos grasos de las muestras de salchicha se observa en la Tabla 3. En esta se evidencia que los tratamiento T3 y T4, formulados con menor contenido de grasa, presentaron el porcentaje más bajo de ácido palmítico. El control aporta el mayor contenido de ácido mirístico, en comparación con los demás tratamientos que muestran una disminución en el contenido de este acido. El tratamiento control presentó un alto contenido de ácido oleico en comparación con los demás tratamientos, presentando en T3 y T4 un mayor contenido de este acido; siendo estos dos tratamientos los que tienen mayor sustitución de PA y menor adición de grasa de cerdo en su formulación. Igual comportamiento presentaron los homólogos (a y y) del ácido linoleico. Estos resultados son similares con los hallazgos de Ayo et al., (2007) quienes remplazaron totalmente la grasa animal de un producto cárnico tipo salchicha y lo compararon con un control, observando que la sustitución total de grasa por nuez aumentó el contenido de ácido oleico de un 11,08\% a un 56,6\%. Se observó que los ácidos grasos eicosapentaenoico (EPA) y docosahexaenoico (DHA) fueron igual para todos los tratamientos, indicando que la incorporación de PA y PS de bovino no tienen influencia en el contenido de estos. De estos resultados se puede concluir que la incorporación de pasta de ajonjolí como sustituto parcial de grasa mejora el perfil lipídico de los tratamientos puesto que el control presentó mayor contenido de ácidos grasos saturados (palmítico y mirístico) en relación con los demás tratamientos, en los cuales aumentó el contenido de los ácidos grasos monoinsaturados y poliinsaturados como el ácido oleico, a-linoléico y $\mathrm{Y}^{-}$ linolénico (Isaza et al., 2010).

Tabla 3: Composición de análisis de perfil de ácidos grasos

\begin{tabular}{|c|c|c|c|c|c|}
\hline \multirow{2}{*}{ Ácido } & \multicolumn{5}{|c|}{ Tratamientos } \\
\cline { 2 - 6 } & $\mathrm{T} 1$ & $\mathrm{~T} 2$ & $\mathrm{~T} 3$ & $\mathrm{~T} 4$ & $\mathrm{~T}$ \\
\hline Mirístico (\%) & $1,39 \pm 0,0005^{\mathrm{b}}$ & $1,36 \pm 0,0015^{\mathrm{b}}$ & $1,18 \pm 0,0015^{\mathrm{a}}$ & $1,20 \pm 0,002^{\mathrm{a}}$ & $1,41 \pm 0,0030^{\mathrm{c}}$ \\
\hline Palmítico (\%) & $25,02 \pm 0,0025^{\mathrm{b}}$ & $25,01 \pm 0,020^{\mathrm{b}}$ & $20,41 \pm 0,0025^{\mathrm{a}}$ & $20.03 \pm 0,0025^{\mathrm{a}}$ & $28,40 \pm 0,0015^{\mathrm{c}}$ \\
\hline Oleico (\%) & $49,47 \pm 0,019^{\mathrm{ca}}$ & $49,53 \pm 0,03^{\mathrm{ca}}$ & $53,82 \pm 0,0442^{\mathrm{b}}$ & $53,88 \pm 0,0311^{\mathrm{b}}$ & $51,49 \pm 0,0496^{\mathrm{a}}$ \\
\hline a-linoleico (\%) & $1,05 \pm 0,0025^{\mathrm{c}}$ & $1,07 \pm 0,0170^{\mathrm{c}}$ & $1,11 \pm 0,0175^{\mathrm{b}}$ & $1,20 \pm 0,0095^{\mathrm{b}}$ & $0,72 \pm 0,0127^{\mathrm{a}}$ \\
\hline Y-linolénico (\%) & $11,00 \pm 0,0081^{\mathrm{ba}}$ & $10,66 \pm 0,012^{\mathrm{ba}}$ & $14,51 \pm 0,0035^{\mathrm{c}}$ & $14,52 \pm 0,0030^{\mathrm{c}}$ & $11,62 \pm 0,0153^{\mathrm{a}}$ \\
\hline EPA (\%) & $<0,0005$ & $<0,0005$ & $<0,0005$ & $<0,0005$ & $<0,0005$ \\
\hline DHA (\%) & $<0,0005$ & $<0,0005$ & $<0,0005$ & $<0,0005$ & $<0,0005$ \\
\hline
\end{tabular}




\section{Rendimiento por cocción}

Los rendimientos por cocción fueron: T1 $(98,86 \pm 0,05)$, T2 $(99,57 \pm 0,05)$, T3 $(99,80 \pm 0,01), T 4(99,16 \pm 0,03)$ y T5 $(98,28 \pm 0,05)$. El tratamiento control fue el que presentó menor rendimiento por cocción, y T3 el que mostró mayor rendimiento por cocción, indicando de esta manera que la incorporación de PS de bovino y PA incide de manera significativa en el rendimiento por cocción. Este comportamiento también se observó en la investigación realizada por Isaza et al., (2010), quienes observaron que a medida que aumentó el nivel de incorporación de PS en el producto cárnico, este presentó un mejor porcentaje de rendimiento. Otros autores han indicado el mismo comportamiento en la elaboración de sus productos cárnicos (Hernández y Güemes, 2010; Barboza et al., 2005).

\section{Análisis de perfil de textura (TPA)}

EI TPA se muestra en la Tabla 4. Los parámetros de dureza y elasticidad de los productos que contenían PA y PS de bovino aumentaron en comparación con el control; indicando que la sustitución parcial de grasa y agua modificó estas características de textura, al igual que los resultados reportados por otros autores (Granados et al., 2013; Hernández y Güemes 2010; Pérez y Vanegas, 2009). En cuanto a la adhesividad, T4 mostró un nivel más alto en comparación con T5, T3 y T2 en los cuales no hubo diferencias significativa $(p<0,05)$.

Se encontraron diferencias estadísticamente significativas $(p<0,05)$ en parámetros como la cohesividad y masticabilidad de los tratamientos, siendo el más cohesivo T1, lo que indico que se requirió mayor fuerza para quebrantar los enlaces de las partículas pertenecientes a la matriz de la salchicha, el T2 y T3 no tuvieron diferencias y el tratamiento control fue el menos cohesivo. La estabilidad obtenida por la pasta de ajonjolí y la ganancia de humedad debido a la incorporación de plasma sanguíneo de bovino suministrado (4\%) formaron una matriz rígida. Por otro lado, a medida que fue aumentando la concentración de plasma sanguíneo y pasta de ajonjolí en las salchichas, estas se volvieron menos masticables, debido al contenido de pasta de pasta de ajonjolí haciendo el interior del alimento más suave. El comportamiento que obtuvo la dureza, la cohesividad y masticabilidad es similar al reportado por Sánchez-Zapata et al., (2011). Estos resultados se obtuvieron debido al contenido de humedad que había en la parte interna de las salchichas, sin embargo se formó una costra debido a la cocción haciéndola más dura por fuera y blanda por dentro.

Tabla 4: Análisis perfil de textura de las salchichas

\begin{tabular}{|c|c|c|c|c|c|c|}
\hline \multirow{2}{*}{ Parámetros } & \multicolumn{5}{|c|}{ Tratamientos } & $\begin{array}{c}\text { Análisis de } \\
\text { Varianza }\end{array}$ \\
\cline { 2 - 6 } & $\mathrm{T} 1$ & $\mathrm{~T} 2$ & $\mathrm{~T} 3$ & $\mathrm{~T} 4$ & $\mathrm{~T}$ & $\mathrm{P}-$ valor \\
\hline Dureza $\left(\mathrm{kg} \mathrm{m} \mathrm{s}^{-2}\right)$ & $53,43 \pm 0,02^{\mathrm{a}}$ & $45,15 \pm 3,61^{\mathrm{b}}$ & $41,20 \pm 1,60^{\mathrm{b}}$ & $45,39 \pm 3,34^{\mathrm{b}}$ & $28,90 \pm 3,80^{\mathrm{c}}$ & 0,0003 \\
\hline Adhesividad $\left(\mathrm{kg} \mathrm{m}^{2} \mathrm{~s}^{-2}\right)$ & $-0,61 \pm 0,15^{\mathrm{b}}$ & $-0,17 \pm 0,05^{\mathrm{a}}$ & $-0,18 \pm 0,06^{\mathrm{ac}}$ & $-0,38 \pm 0,08^{\mathrm{ab}}$ & $-0,18 \pm 0,08^{\mathrm{a}}$ & 0,0117 \\
\hline $\begin{array}{c}\text { Cohesividad } \\
\text { (adimensional) }\end{array}$ & $0,42 \pm 0,03^{\mathrm{a}}$ & $0,35 \pm 0,10^{\mathrm{b}}$ & $0,28 \pm 0,25^{\mathrm{c}}$ & $0,35 \pm 1,5^{\mathrm{b}}$ & $0,26 \pm 1,86^{\mathrm{c}}$ & 0,0024 \\
\hline $\begin{array}{c}\text { Elasticidad } \\
\text { (adimensional) }\end{array}$ & $1,15 \pm 0,06^{\mathrm{a}}$ & $0,96 \pm 0,03^{\mathrm{b}}$ & $0,98 \pm 0,03^{\mathrm{b}}$ & $1,04 \pm 0,08^{\mathrm{ab}}$ & $0,95 \pm 0,01^{\mathrm{b}}$ & 0,0029 \\
\hline Masticabilidad $(\mathrm{kg})$ & $25,81 \pm 0,25^{\mathrm{a}}$ & $15,17 \pm 0,50^{\mathrm{b}}$ & $11,31 \pm 0,13^{\mathrm{c}}$ & $15,58 \pm 0,2^{\mathrm{b}}$ & $7,14 \pm 0,29^{\mathrm{c}}$ & 0,0035 \\
\hline
\end{tabular}

\section{Aceptabilidad}

La aceptabilidad para cada tratamiento fue el siguiente: T1 $(3,37 \pm 1,39)$, T2 $(4,05 \pm 0,90)$, T3 $(3,55 \pm 01,46)$, T4 $(3,30 \pm 1,18)$ y T5 $(4,00 \pm 1,32)$. El tratamiento T2 obtuvo mayor aceptabilidad que los demás, posiblemente debido a que fue el tratamiento que menor porcentaje de sustituto de grasa y agua se le adicionó, conservando características organolépticas muy parecidas a la de una salchicha normal como la de control. Hubo grandes diferencias significativas $(p<0,05)$ en el tratamiento T1, T3 y T4 en comparación con T2 y T5 (control). En otros estudios Márquez et al., (2006) estudiaron las propiedades funcionales del plasma; en este caso formularon un embutido con agregado de piel de pollo emulsificada con sangre de bovino, se formularon tres productos (A, B y C), el producto $\mathrm{C}$ (control) a los tres productos se les determinó la aceptabilidad, indicando que no hubo diferencias ( $p>0,05$ ) en la aceptabilidad de los productos. Ahmed et al., (1990), analizaron la aceptabilidad de salchichas de cerdos bajas en grasa, las cuales fueron formuladas para reemplazar la grasa animal con la adición de agua, reportando una buena aceptabilidad en las salchichas. Considerando como patrón de aceptabilidad una puntuación igual o superior a cuatro, el tratamiento T2 (1,5\% PA y $2 \%$ PS) fue aceptado por el $67,50 \%$ de los panelistas, mientras que el control obtuvo un $62,50 \%$ de aceptabilidad como se observa en la Figura 1. 


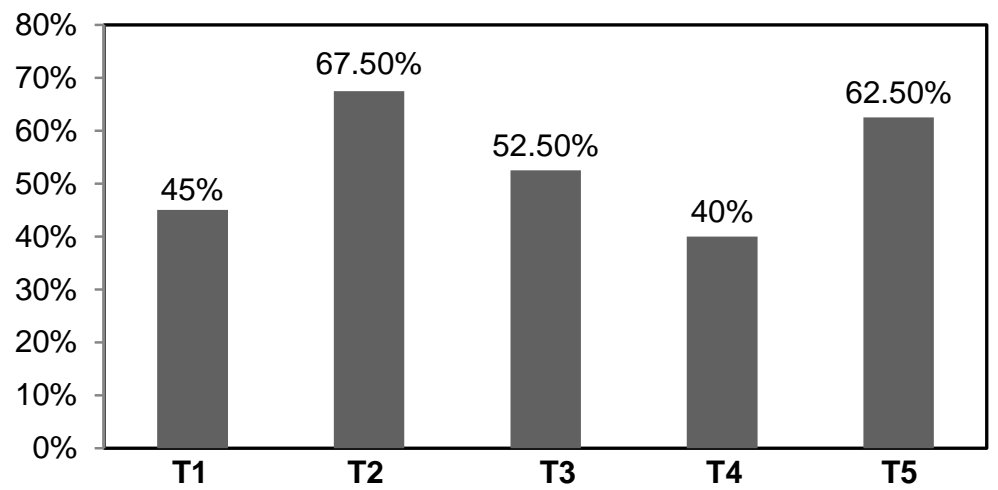

Fig. 1: Clasificación de panelista con puntajes igual o superior a 4 en aceptabilidad

\section{CONCLUSIONES}

La sustitución parcial de grasa por pasta de ajonjolí y de agua por plasma sanguíneo de bovino en la elaboración de un producto cárnico tipo salchicha disminuyó el contenido graso y aumentó el contenido de proteína, obteniendo un producto cárnico más saludable, con mayor contenido de humedad y por consiguiente con un buen rendimiento por cocción. Se mejoró el perfil lipídico, sin embargo no produjo cambio en la composición de los ácidos grasos eicosapentaenoico (EPA) y docosahexaenoico (DHA), ya que fue igual para todos los tratamientos. Los parámetros de texturas se vieron afectados con la adición de PA y PS de bovino. Es posible incorporar pasta de ajonjolí y plasma sanguíneo de bovino en concentraciones de 1,5\% A y $2 \%$ PS en la formulación de un producto cárnico, sin que ello tenga un efecto negativo en la aceptabilidad.

\section{AGRADECIMIENTOS}

Los autores agradecen a COLCIENCIAS por la financiación del proyecto "Desarrollo de productos de pastelería empleando como base pasta de ajonjolí (Sesamum indicum) y plasma sanguíneo de bovino como surfactante" en la Convocatoria 664 "CONVOCTORIA PARA EL APOYO A LA INNOVACIÓN EN GASTRONOMÍA - 2014" de donde se derivan los resultados de este artículo.

\section{REFERENCIAS}

A.O.A.C., Association of Official Analytical Chemists, Official Methods of Analysis of AOAC International, 17th edition, Maryland, USA (2003)

Acevedo, D., P.M. Montero y Y.A. Marrugo, Caracterización Reológica de Pastas untuosas Artesanal y Tecnificada de Ajonjolí (Sesamum indicum) Cultivado en Zambrano-Bolívar (Colombia), Información Tecnológica, 25(4), 73-78 (2014)

Acevedo, D., Y. Marrugo y P. Montero, Evaluación de las propiedades reológicas de pastas de ajonjolí artesanal y tecnificada, Rev. U.D.C.A Act \& Div. Cient., 16(1), 245-251 (2013)

Ahmed, P.O. y otros cuatro autores, Physical and sensory characteristics of low fat fresh pork sausage processed with various levels or added water, Journal of Food Science, 55(3), 625-628 (1990)

Ayo, J. y otros cinco autores, Effect of total replacement of pork backfat with walnut on the nutritional profile of frankfurters, Meat Science, 77(2), 173-181 (2007)

Barboza, Y. y otros cuatro autores, Utilización del plasma sanguíneo de bovino como fuente proteica en la formulación de un medio de cultivo pata lactobacilos, Revista Científica Facultad de Ciencias Veterinarias, 4, 5559 (1994)

Benítez, B. y otros cuatro autores, Calidad nutricional y aceptabilidad de un producto formulado con carne de pollo deshuesada mecánicamente, plasma y glóbulos rojos de bovino, ALAN, 52(3), 307-312 (2002) 
Benítez, B. y otros seis autores, Calidad microbiológica de una galleta formulada a base de harina de yuca y plasma de bovino, Rev. Fac. Agron. U. Zulia, 28(2), 260-272 (2011)

Camacho, A., P.M. Montero y M. Duran, Efecto de la adición de plasma sanguíneo de bovino en el contenido protéico, aceptabilidad y calidad microbiológica de una bebida a base de arroz, Revista Alimentos Hoy, 22(31), 104-108 (2014)

Chiriboga, M. G., Evaluación de la efectividad nutricional de la pasta de ajonjolí (Sesamum indicum L.) como sustituto de la pasta de soya en el crecimiento de codornices (Coturnix coturnix), Tesis de pregrado, Ingeniería de Agroempresas, Universidad Francisco de quito, Colegio de Ciencias e Ingenieria, Quito, Ecuador (2013)

Decker, E.A. y Y. Park, Healthier meat products as functional foods, Meat Science, 86(1), 49-55 (2010)

Feng, T. y otros siete autores, Physicochemical properties and sensory evaluation of Mesona Blumes gum/rice starch mixed gels as fat-substitutes in Chinese Cantonese-style sausage, Food Research International, 50(1), 85-93 (2013)

Figueroa, O., J. Zapata y G. Gutiérrez, Modelamiento de la cinética de hidrólisis enzimática de proteínas del plasma bovino, Revista EIA, 9(17), 71-84 (2012)

García, O.O., J. Ruiz y I. Acevedo, Evaluación físico-química de carnes para hamburguesas bajas en grasas con inclusión de harina de quinchoncho (Cajanus Cajan) como extensor, Revista Científica, 22(6), 497-506 (2012)

Gok, V. y otros tres autores, Effect of ground poppy seed as a fat replacer on meat burgers, Meat Science, 89, 400-404 (2011)

Gómez, L., O. Figueroa y J. Zapata, Actividad antioxidante de hidrolizados enzimáticos de plasma bovino obtenidos por efecto de Alcalasa® 2.4 L, Información Tecnológica, 24(1), 33-42 (2013)

Granados, C., L.E. Guzmán y D. Acevedo, Análisis proximal, sensorial y de textura de salchichas elaboradas con subproductos de la industria procesadora de atún (Scombridae thunnus), Información tecnológica, 24(6), 29-34 (2013)

Hernández, S. y N. Güemes, Efecto de la adición de harina de cáscara de naranja sobre las propiedades fisicoquímicas, texturales y sensoriales de salchichas cocidas, NACAMEH, 4(1), 23-36 (2010)

Hleap, J. y A.V. Velasco, Parámetros fisicoquímicos durante el almacenamiento de salchichas elaboradas a partir de tilapia roja (Oreochromis sp.), Revista de la Facultad de Ciencias Agropecuarias: 10(1), $42-50$ (2012)

Hurtado, S. y otros seis autores, Use of porcine blood plasma in "phosphate-free frankfurters", Procedia Food Science, 1, 477-482 (2011)

Isaza, J. y otros cuatro autores, Producción y propiedades funcionales de plasma bovino hidratado en embutido tipo salchichón, Revista Colombiana de Ciencias Pecuarias, 23(2), 199-206 (2010)

Isaza, J., y otros cuatro autores, Producción y propiedades funcionales de plasma bovino hidratado en embutido tipo salchichón, Rev. Ciencias Pecuarias, 23(2), 199-206 (2010)

Julio, L.C., P.M. Montero y D. Acevedo, Calidad y aceptabilidad de chorizos formulados con plasma sanguíneo bovino y pasta de ajonjolí, Información Tecnológica, 26(3), 25-32 (2015)

Márquez, E. y otros cinco autores, Formulación de un embutido con agregado de piel de pollo emulsificada con sangre de bovino, Revista Científica FCV-LUZ, 16(4), 438-444 (2006)

Parés, D., E. Saguer y C. Carretero, 9 - Blood by-products as ingredients in processed meat, Processed Meats, Improving Safety, Nutrition and Quality, A volume in Woodhead Publishing Series in Food Science, Technology and Nutrition, 218-242 (2011) 
Perez, D. y O. Vanegas, Sustitución parcial de la grasa de cerdo por grasas de palma en una pasta fina cárnica modelo, Ciencia y Tecnología de Alimentos, 19(1), $52-55$ (2009)

Piñero, C. y otros seis autores, Atributos sensoriales y químicos de un producto cárnico ligero formulado con fibra soluble de avena, Revista Científica de América Latina, 15(3) 279-285 (2004)

Rodríguez, L., A. Pérez y M. Campderrós, Plasma bovino tecnológicamente mejorado para aplicaciones alimenticias, La Alimentación Latinoamericana, 93, 44-48 (2011)

Ruiz, D. y E. Pacheco, Utilización de inulina y carragenina en la elaboración de salchichas de carne bajas en grasa, Revista de la Facultad de Agronomía LUZ, 33(3), 165-178 (2007)

Schmiele, M. y otros cuatro autores, Dietary fiber as fat substitute in emulsified and cooked meat model system, LWT - Food Science and Technology, 61(1), 105-111 (2015)

Tirado, D.F., D. Acevedo y L.E. Guzmán, Coeficientes convectivos de transferencia de calor durante el freído de láminas de tilapia "Oreochromis niloticus", Información Tecnológica, 24(6), 41-46 (2013)

Zamorano, M., E. Guzmán y E. Meléndez, Composición proximal y perfil de ácidos grasos de combos de hot dogs consumidos en cadenas de comidas rápidas en la región metropolitana de chile, Revista Chilena de Nutrición, 34(2), 150-155 (2007) 
